\title{
Learning Effectiveness of Lecture Versus Laboratory: are labs worth it?
}

\author{
By B. Hufnagel ${ }^{1,2}$, E. Loh ${ }^{1}$ \& J. Parker ${ }^{2,3}$ \\ ${ }^{1}$ Department of Physics and Astronomy, ${ }^{2}$ Division of Science Education, ${ }^{3}$ Department of \\ Teacher Education, Michigan State University, East Lansing, MI USA
}

\section{Introduction}

Michigan State University (MSU) serves a large and diverse student population, $\sim 1000$ of whom take the astronomy course for non- science majors each year. Significant resources are also invested in the related astronomy lab, enrolling about half the lecture students. Although this lab is optional, the students are required to complete one lab course for their degree. In the fall of 1995, we undertook an extensive assessment of student learning in these astronomy courses.

\section{The Student Population}

Unlilke most astronomy research, information about the entire population under study (403 students) was available. This included name, major, grade earned, and concurrent enrollment in lab and lecture. Fig. 1(a) shows that the shapes of the grade distributions differ for the day and evening classes, and that neither is Gaussian. Therefore the day and evening classes will be analysed separately, and statistics such as mean and standard deviation are good descriptors for only the day-class students receiving a 1.0 lecture grade or above. The lab grades were also plotted for the day and evening classes separately, and no difference in the shapes of the distributions were apparent (Fig. 1(b)). This indicates that the different grade distributions of the day and evening lectures are lecture-dependent, rather than rooted in the nature of the students taking day versus evening classes. The lecture and lab grades were also plotted for males versus females (gender information was not available for eleven students), and no gender bias was evident.

One way to assess the effectiveness of the lab curriculum is to determine if those students taking the lab plus lecture concurrently have higher grades in the lecture than students taking only the lecture. Figs. 2 and 3 show that the lecture grades for students talking the lab are better. However, this is statistically significant for the women in the day lecture (Pearson's $r=0.34$ ), but not so for the men (Pearson's $r=0.94$ ) (see Press et al. 1986 for a discussion of Pearson's Coefficient of Correlation $r$ ). Another test of effectiveness is to look for a correlation between a high grade in lab and a high grade in lecture. A student's lab grade was found to correlate weakly with his or her lecture grade, with a Pearson's $r$ value of $\sim 0.51$ for both sexes.

The population statistics can also test the hypothesis that a computer-intensive lab course is gender biased. No gender bias either in avoidance of the lab by women, in retention of women in the lab course, or in lab grades earned by women was evident. There were $48 \%$ and $50 \%$ women lab students at the beginning and end of the semester, respectively.

\section{Introduction to the Survey}

The assessment instrument for this fall 1995 study was an entrance and exit survey developed by us to test the explicit goals of the lab course. Of the 403 students enrolled in three lecture sections, 271 entrance and 192 exit surveys were obtained. About half of these students also enrolled for the optional lab course. Anonymity of the students encouraged candid feedback, and the entrance surveys were administered by faculty other than the assigned professor.

The survey consisted of two parts, the first being background questions, and the second twelve 
Population Grades: Day versus Evening Classes
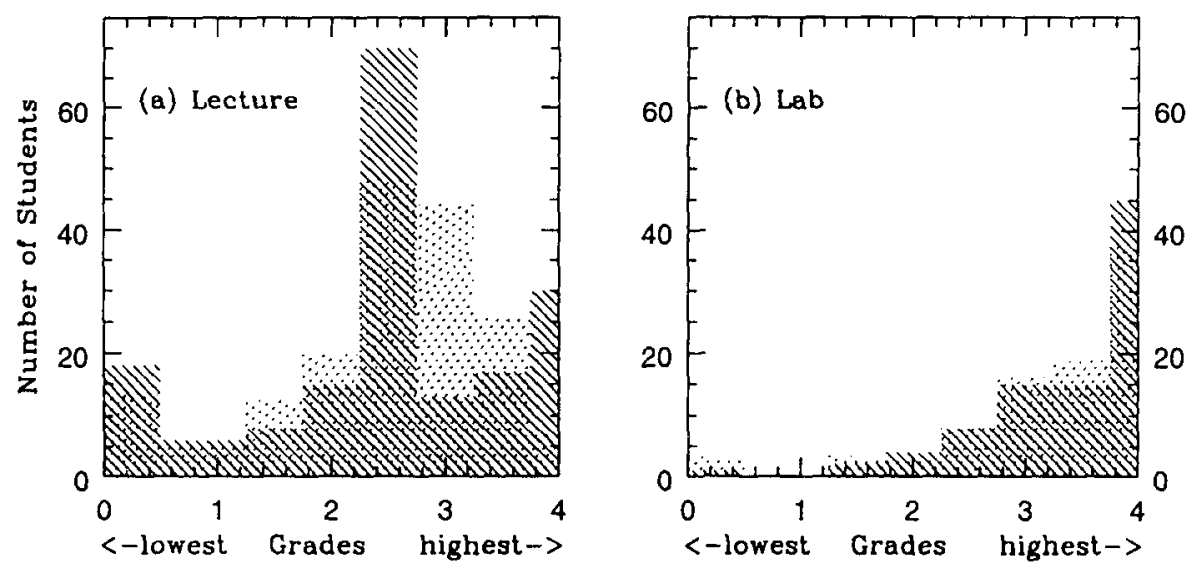

FIgURE 1. The distribution of grades for day (lighter shade) and evening (darker shade) (a) lecture and (b) lab classes. The day-lecture data have been scaled by 0.80 and 0.67 (lecture and lab, respectively), to match the smaller evening-class size. The grades for the day and evening lecture classes are distributed differently, and none of the four are Gaussian.

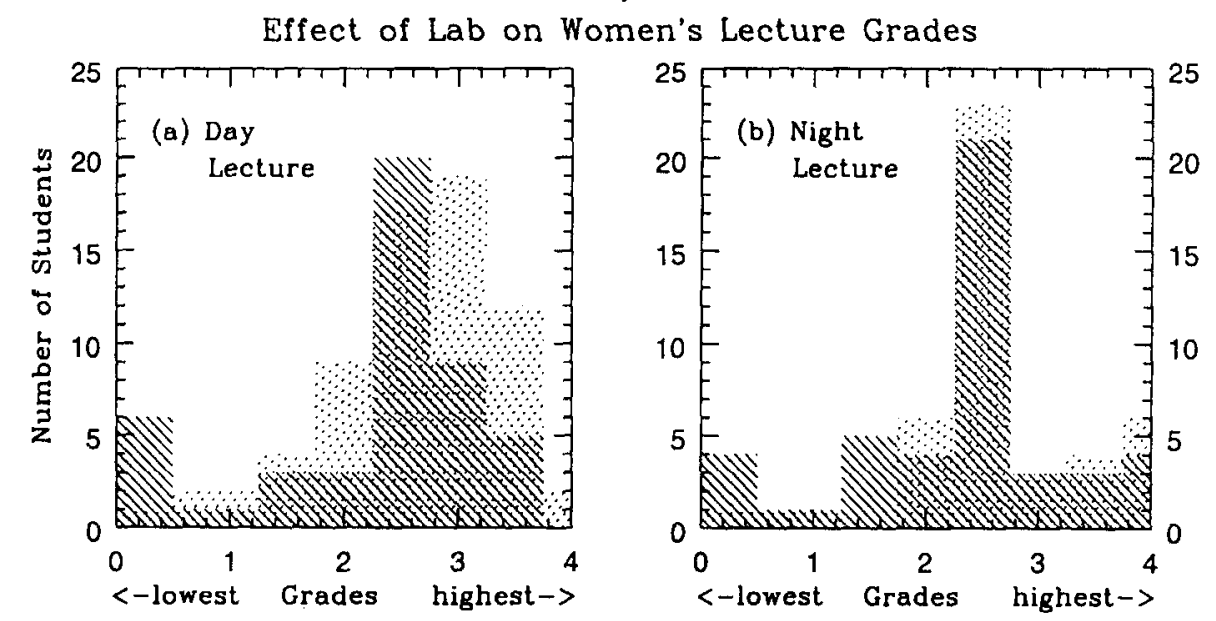

Figure 2. Comparison of lecture grades for women taking both lecture and lab versus those taking the lecture only. Panel (a) is the day-lecture grades, and Panel (b) the night-lecture grades. The dark- shaded histograms are grades for women taking the lecture only, and the light ones for women taking both the lab and lecture.

content questions. The twelve questions had two parts, the first a bimodal (e.g., yes/no) choice, and the second an open-ended essay question probing the student's level of understanding.

A concern with any sample is if it is representative of the population. The entrance survey included more women than men (58 and $40 \%$ respectively), while the exit survey was representative of the population's almost even split. Other questions, however, such as math background and why they chose astronomy rather than another science course, were answered in similar percentages in both the exit and entrance surveys. 


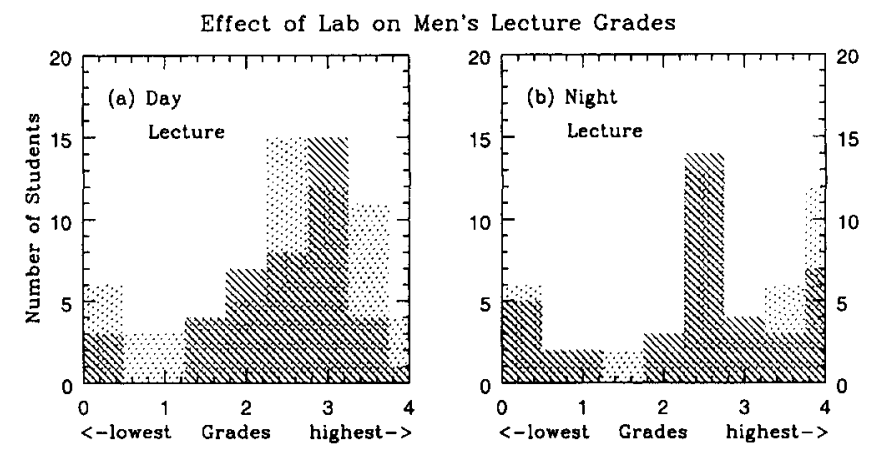

FigURE 3. Comparison of lecture grades for men taking both lecture and lab versus those taking the lecture only. The dark-shaded histograms are grades for men taking the lecture only, and the light ones for men taking both the lab and lecture.

\section{The Survey Results}

Was the lab more effective than the lecture? This depended on the lab. For example, the lab on variable stars was less successful in changing a widely-held $(\sim 75 \%)$ misconception that space-based observing is necessary to observe variable stars, as $59 \%$ of lab students vs. $50 \%$ of non-lab students still held the misconception at the end of the course. (Only a handful of students were willing to explain their answers.) However, the second lab on measuring mass using dynamics shifted the lab students' partial understanding to a complete one $(71 \%$ of lab students). The non-lab students' understanding at the end of the course resembled that of the lab students after the first dynamical-mass measurement lab. Almost all of the students began the course knowing that it was possible to measure mass dynamically, but the lab students where able to explain how to do it.

Did the lab course enhance retention of knowledge? The "entrance"survey was given after several of the labs had already been taught. The two surveys then measured, for these four labs, whether the students retained the knowledge to the end of the semester. The results are mixed, with the lab-only students doing better in retention on some labs but worse than the non-lab students on others.

Are the students taking lab different from those taking only the lecture? Both lab and lecture students gave "The course sounded interesting" as their most common reason (45\%) to choose astronomy. Slightly more lab students expressed a prior interest in astronomy (28\%) versus those not taking the lab (20\%). The maths backgrounds were similar, but the most significant difference was that lab students were far more likely to be first-year students (40\% vs. $14 \%$ ).

For the exit surveys, we added an open-ended question about the source of their knowledge of astronomy. The most popular response was television (53\%), followed by school (33\%).

\section{Discussion}

When the title of a paper is phrased as a question, the answer is usually "No." However, in this case, the answer to the question "Learning effectiveness of lecture versus lab: are labs worth it?" is "It depends."It depends on the lab itself, the gender of the student, and how it inter-relates with the lecture. For example, the second lab on the same topic, such as our two labs on dynamical mass, shifted the typical partial understanding to a complete one. Although lecture grades were in general better for students taking the lab and lecture concurrcutly, only for women in the 250-student day class was the difference statistically significant. We suggest that this is more strongly lecturer- than student-dependent, though it is possible that women in extremely large classes respond particularly well to the cooperative learning format of our labs.

The question as to where students get their understanding of astronomy is an interesting one. We intend to explore this further as guidance for our community outreach efforts. 
We recommend that an institution considering changes to its astronomy lab curriculum first define the purpose of the lab. For example, is the lab intended to complement the lecture course with active learning of the same topics? Alternatively, a lab course might be designed to add breadth, such as hands-on use of the analytical tools used by astronomers or familiarity with the night sky. If the lab is meant to complement the lecture, the second step is to compile population statistics to test if this purpose is already being met. This can inexpensively answer the question posed by this paper for current and past lab courses, identify gender or other biases, and discriminate between the need for drastic change in the curriculum versus maintenance. A student survey can then be designed to further investigate which labs work and which don't, to better understand the student population, or to assess the effectiveness of changes as they are introduced.

\section{Acknowledgments}

We appreciate the cooperation of the two instructing faculties and their students who cheerfully participated in the survey. We thank the teaching assistants, and also R. Wilhelm for his comments on this paper. $\mathrm{BH}$ also thanks $\mathrm{C}$. Suelter for conceiving of and promoting her postdoctoral position in astronomy and science education research.

\section{REFERENCES}

Press, W.J., Flannery, B.P., Teukolsky, S.A., \& Vetterling, W.T., (1986) Numerical Recipes, New York: Cambridge University Press, 630. 